\title{
The Role Of Fibroscan In Detecting Hepatic Fibrosis Induced By Methotrexate
}

University "Ovidius" of Constanta, Faculty of Medicine

\begin{abstract}
Introduction: Liver represents the main place of drug metabolisation. Drugs and toxic substances reach the level of liver after absorption at gastro-intestinal level. Drug hepatotoxicity represents an important chapter of iatrogenic pathology, because the hepatic lesions induced by drugs include extremely diverse clinical, biological and histological expressions that can take the aspect of any form of acute or chronic hepatobiliary disease. Hepatic lesions induced by drugs (LHIM) represent a histological and/or biochemical alteration caused and attributed to the consumption of a drug. Hepatic elastography (Fibroscan) represents a noninvasive method of quantification of hepatic fibrosis.

Material and method: We carried out a retrospective study and longitudinally prospective study that included a set of patients under treatment with Methotrexate, amounting to 76 , divided into 2 subsets: a subset consisting of patients with hepatitis to Methotrexate, subset 1 , of 23 patients, a subset of patients under treatment with Methotrexate but with normal hepatic samples, subset $b$, of 53 patients.

Results: We carried out fibroscan a $t$ a $11 \mathrm{t}$ he patients treated with Methotrexate, obtaining a medium score of fibrosis of 6.23 for the subset a with hepatitis at Methotrexate and of 5.33 for the subset $b$ with normal hepatic samples. We made a correlation between the cumulated dose of Methotrexate and the
\end{abstract}

\section{Relu Liviu Craciun}

Faculty of Medicine, Constanta, Romania

1st University Alee, Camp B

email : rlcraciun@yahoo.com change of hepatic samples.

Conclusions: There is a significant correlation between hepatic fibrosis induced by Methotrexate and the cumulated dose of Methotrexate. The possibility of utilization of fibroscan which is a completely painless method, reproducible, for the monitoring of the treatment with Methotrexate both at patients with changed values of the hepatic tests as weir as those with normal values must be considered.

Keywords: methotrexate, hepatic fibrosis, fibroscan

\section{Introduction}

Hepatic lesions induced by drugs (LHIM) represent a histological and/or biochemical alteration caused and attributed to the consumption of a drug. Drug hepatotoxicity represents an important chapter of iatrogenic pathology, because the hepatic lesions induced by drugs include extremely diverse clinical, biological and histological expressions that can take the aspect of any form of acute or chronic hepatobiliary disease [1,2].

The hepatotoxic effects caused by drugs were described starting from the $19^{\text {th }}$ century - hepatic steatosis caused by phosphorus and hepatotoxicity of tannic acid.

In the last decades a 11 time increase of LHIM is reported, due to the increase of the number 1 of used drugs but also by their more frequent recognition [3].

The high number of chronic hepatic disorders that evolve towards hepatic fibrosis requires the creation of some new tracking and stadialization 
methods in medical practice.

The fibrosis, which leads to various morphologic, hemodynamic and functional changes at liver level, the result being the occurrence of some quantifi able changes $[4,5,6]$.

The objectives of hepatic fibrosis are:

- demonstration of lesions and their quantification which allows the making of some therapeutic decisions

- $\quad$ surveillance of the evolution of fibrosis under treatment

- screening for hepatopathies with fibrosing potential.

Hepatic biopsy still represents "golden standard" for the evaluation of steatosis and hepatic

fibrosis but it is an invasive method, difficult to accept by the patients, being also accompanied by side effects. Thus, at world level, the largest part of the modem researches was directed towards the implementation of some alternative non-invasive diagnosis methods that provide sufficient accuracy so as to allow both a precise diagnosis as well as to provide the possibility of monitoring the evolution of hepatopathy by rehabilitation and reproductivity $[7,8]$. The most frequent adverse effects of small doses of Methotrexate administered for a long time for immunosuppressive purposes as it happens in rheumatoid arthritis, psoriasis, psoriatic arthropathy, some forms of ankylosing spondylitis, can cause hepatic fibrosis with relatively increased incidence.

\section{Material and method}

We carried out a retrospective study and longitudinally prospective study that included a set of patients under treatment with Methotrexate, amounting to 76 , divided into 2 subsets: a subset consisting of patients with hepatitis to Methotrexate, subset 1, of 23 patients, a subset of patients under treatment with Methotrexate but with normal hepatic samples, subset $b$, of 53 patients.

Inclusion criteria:
- $\quad$ patients under treatment with Methotrexate

- $\quad$ AgHBs and Ac anti VHC negative

- $\quad$ ALAT increase (alanine aminotransferase) $>$ $2 \mathrm{~N}$ and/or

- $\quad$ FAS increase $>2 \mathrm{~N}$ for subset a

- $\quad$ normal hepatic samples for subset $b$

\section{Results}

1) Distribution of the patients according to the disorder for which they have received MTX

Table I Distribution of the patients according to the disorder for which they have received MTX

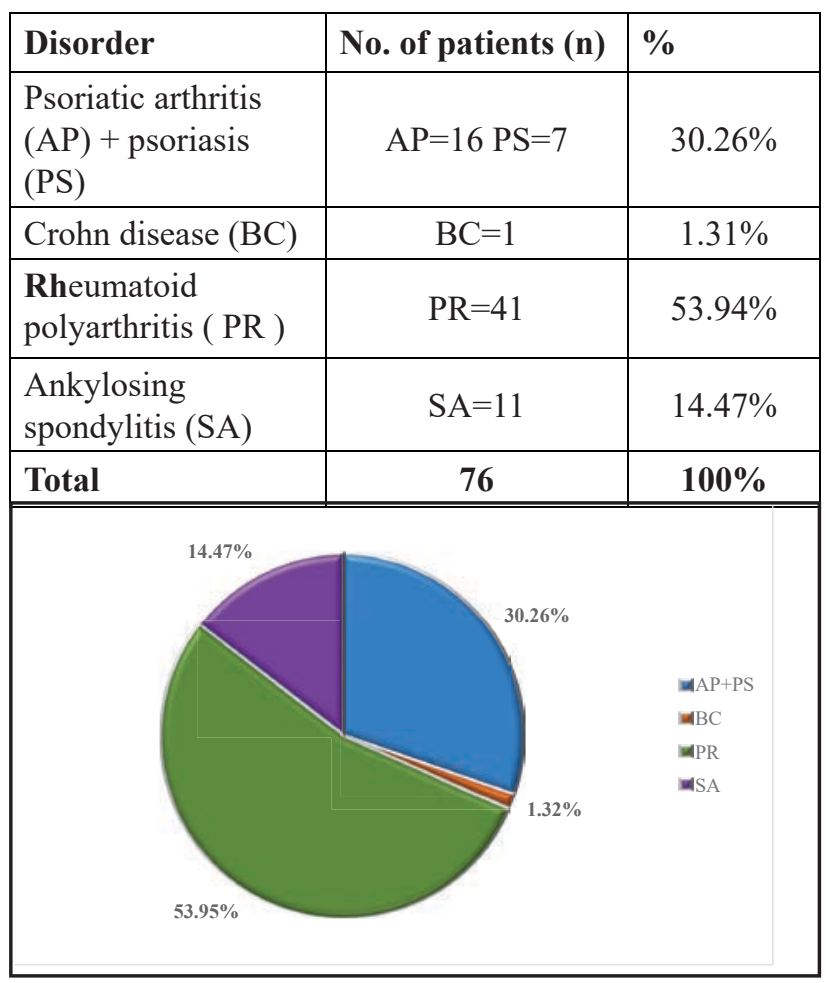

Figure 1 Distribution of the patients according to the disorder for which they have received MTX

Most of the patients included in the study were with rheumatoid arthritis 53.94\% and with Crohn 
disease $1,31 \%$.

2) Structure of the 2 subsets:

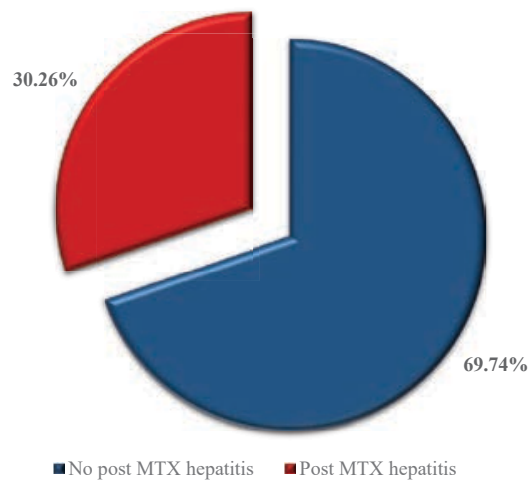

Figure 2 Structure of the two subsets

$30.26 \%$ of the patients under treatment with Methotrexate had increases of transaminases $>2 \mathrm{~N}$ and /or FAS $>2 \mathrm{~N}$.

3) Distribution of the patients of the two sets according to the medium fibrosis score (fibroscan): In our study we performed fibroscan at all the patients treated with Methotrexate, achieving a medium fi brosis score of 6.23 for the subset a with hepatitis at Methotrexate and of 5.33 for the subset $b$ with normal hepatic samples.

Table II Descriptive statistics

\begin{tabular}{|l|c|c|}
\hline & $\begin{array}{l}\text { No postMTX } \\
\text { hepatitis }\end{array}$ & $\begin{array}{l}\text { Post MTX } \\
\text { hepatitis }\end{array}$ \\
\hline Range & $3-8.20$ & $3.60-9.70$ \\
\hline Number of values & 53 & 23 \\
\hline Minimum & 3 & 3.60 \\
\hline Q1 (25\%) & 4.20 & 4.50 \\
\hline Median & 5.10 & 5.60 \\
\hline Q3 (75\% ) & 6.40 & 7.90 \\
\hline Maximum & 8.20 & 9.70 \\
\hline $\begin{array}{l}\text { Weighted average } \\
\text { (Mp) with CI 95\% }\end{array}$ & 5.33 & 6.23 \\
\hline Standard deviation & \pm 1.39 & \pm 1.86 \\
\hline $\begin{array}{l}\text { Standard error of } \\
\text { the average (SEM) }\end{array}$ & 0.19 & 0.8 \\
\hline $\begin{array}{l}\text { Variation } \\
\text { coefficient }\end{array}$ & $26.9 \%$ & $29.96 \%$ \\
\hline
\end{tabular}

\section{Conclusions}

1. The most frequent adverse effects of small doses of Methotrexate administered for a long time for immunosuppressive purposes as it happens in rheumatoid arthritis, psoriasis, psoriatic arthropathy, some forms of ankylosing spondylitis, can cause hepatic fibrosis with relatively increased incidence.

2. Hepatic toxicity occurs after a long utilization of the treatment with Methotrexate.

3. Alcohol consumption, antecedents of infection with $\mathbf{B}$ or $\mathrm{C}$ hepatitis virus, obesity, sugar diabetes represent risk factors for hepatic fibrosis.

4. There is a significant correlation between hepatic fibrosis induced by Methotrexate and the cumulated dose of Methotrexate. The utilization of fibroscan in monitoring treatment with Methotrexate should become a routine at all the patients for avoiding hepatic complications respectively hepatitis or hepatic cirrhosis, the method being completely painless, reproducible, with a possibility of being performed both at patients with changed values of the hepatic tests as well as those with normal values.

\section{References}

1. Bohan, A. \& Boyer, J.L. (2002). Mechanisms of hepatic transport of drugs: implications for cholestatic drug reactions. Semin Liver Dis, 22(2), 123-136. doi: 10.1055/s-2002-30099

2. Dumitrascu D. (1997). Hepatic lesions induced by drugs.. In M. Grigorescu \& O. Pascu (Eds), Clinical gastroenterology treaty, vol II (pp. 283297). Bucharest : Technical Publishing House,. 
3. Farrel, G.C. (1995). Importance of drug-induced liver disease. In: G.C. Farrel (ed), Drug-Induced Liver Diseases (pp 29-41), Edinburgh: Churchill Livingstone.

4. Hagenbuch, B. \& Meier, P.J. (2004). Organic anion transporting polypeptides of the OATP/ SLC21 family: phylogenetic classification as OATP/ SLCO superfamily, new nomenclature and molecular/functional properties. Pflugers Arch, 447(5), 653-665. doi: 10.1007/s00424003-1168-y

5. Keppler, D. \& Konig, J. (1997). Hepatic canalicular membrane 5: Expression and localization of the conjugate export pump encoded by the MRP2 (cMRP/cMOAT) gene in liver. FASEB J, 11(7), 509-516.

6. Keppler, D. \& Konig, J. (2000). Hepatic secretion of conjugated drugs and endogenous substances. Semin Liver Dis, 20(3), 265-272. doi: 10.1055/s2000-9391

7. Lecureur, V., Courtois, A., Payen, L., Verhnet, L., Guillouzo, A. \& Fardel, O. (2000). Expression and regulation of hepatic drug and bile acid transporters. Toxicology, 153(1-3), 203-219.

8. Lee, J. \& Boyer, J.L. (2000). Molecular alterations in hepatocyte transport mechanisms in acquired cholestatic liver disorders. Semin Liver Dis, 20(3), 373-384. doi: 10.1055/s-20009390 